The father was recorded as the parent to be experience most Domestic Violence; $40 \%$. Other family members who experienced domestic violence ranged from the mother $32 \%$, brother $12 \%$ and sister $8 \%$. Other family members were $8 \%$.

$100 \%$ of the referrals did not report the domestic violence in the carer relationships, nor did referring agencies recorded safeguarding adults concerns.

Conclusion. The findings from this audit raises a couple of clinical, legal and safeguarding adults work in National Forensic Mental Health Services with regards to family work. Firstly, the need to (re)conceptualising family work in the context of trauma informed care. Secondly, family work should offer some families, who are victim of crime, a restorative approach. Thirdly, safeguarding adults will need to consider complex caring relationships and acknowledged this as part of care planning and support.

\section{Has the COVID-19 pandemic affected out-of-hours presentations in CAMHS?}

\section{Sacha Evans}

Mildred Creak Unit, Great Ormond Street Hospital

doi: 10.1192/bjo.2021.845

Aims. The aim of this project was to look at whether the COVID-19 pandemic, specifically lockdown, has impacted out-of-hours presentations to Child and Adolescent Mental Health Services (CAMHS) in North Central and East London.

Method. Specialist Registrars (SpRs) on the Royal London/Great Ormond Street CAMHS Higher Training Scheme are contacted for advice regarding all CAMHS presentations in the North Central and East London area. Responsibilities includes provision of advice to 6 hospitals (including 4 emergency departments) and 4 child and adolescent inpatient units. A record of all phone calls and call-outs, including Mental Health Act and Section 136 (S136) assessments are maintained and this study compares pre- and post-COVID-19 data to see if there are any differences in number of presentations, on-site assessments (including Mental Health Act and S136 assessments over 2019 and 2020.

Result. Numbers of CAMHS presentations were lower in 2020 (mean 74 patients per month) compared with 2019 (60 patients per month). This was consistent across all months except October and December. The largest difference was seen in March: 109 patients presented in March 2019, compared with 55 in March 2020. This is also reflected in the number of assessments conducted on site. However, there do not appear to be differences in the numbers of Mental Health Act or S136 assessments undertaken over 2020, compared with 2019.

Conclusion. CAMHS out-of-hours presentations dropped off significantly at the start of the COVID-19 pandemic in the UK, and in particular, with the first lockdown (March to July 2020). Specialist Registrars provided advice via telephone less frequently in 2020 compared with 2019, and were required to do fewer on-site assessments of children and young people presenting with mental health difficulties.

There were no significant differences in Mental Health Act or S136 assessments between the two years, however, these numbers are too small to make any meaningful conclusions.

It is likely that children and adolescents were less likely to present to emergency departments for assessment of their mental health difficulties during the COVID-19 pandemic, rather than this reflecting a true reduction in mental health difficulties.

Recommendations:

It is helpful to continue to monitor CAMHS out-of-hour presentations.
Trusts may want to consider alternative settings for providing emergency CAMHS assessments, for example, mental health hubs. Limitations:

This provision of data is subject to recall bias.

Service evaluation project: the effects of healthy weight commissioning for quality and innovation (CQUIN) interventions on metabolic parametrs of service users in a medium secure forensic ward

Elena Fage ${ }^{1 *}$ and Gaurav Sharma ${ }^{2}$

${ }^{1}$ Brent Home Treatment Team and ${ }^{2}$ St Bernards Hospital West London NHS Trust

${ }^{\star}$ Corresponding author.

doi: 10.1192/bjo.2021.846

Aims. Service users of secure forensic units can be prone to weight gain due to various reasons including medications, physical illnesses, sedentary habits and mental health difficulties. They in turn are at greater risk of obesity related health problems like Diabetes, Hypercholesterolaemia, Ischaemic Heart Disease, Depression among others.

Our project was aligned with government's plan to improve prevention and screening for the obesity and metabolic syndrome among the patients of medium secure facilities by 2020 .

Our primary objective was to gather and analyse the data on current metabolic parameters such as weight, body mass index (BMI), blood pressure (BP) and biochemistry markers of the service users on a 18 bedded Male medium secure long term rehabilitation ward.

Our secondary objective was to suggest healthy weight interventions that would help patients to loose weight and to explore the effects of these interventions on biochemistry markers and vital signs parameters.

Method. We collected cross sectional data in given period of time (mid-March 2020). Seventeen service users were included in the final sample. Following initial data collection, we suggested various healthy weight interventions for the patients and repeated data collection after four months (July 2020).

Interventions offered:

Healthy eating group

1:1 sessions with doctors and pharmacists

Gym referrals

Dietician referrals

Relaxation group

Result. Baseline

Fourteen patients on the ward $(n=14)$ were found to be either overweight or obese. Two patients $(n=2)$ had high BP, twelve patients $(n=12)$ had deranged lipid profile, six $(n=6)$ had high blood glucose (existing Diabetes).

Following intervention

Fourteen patients $(n=14)$ remained either overweight or obese. Nine patients $(n=9)$ lost weight following the intervention. Eight patients $(n=8)$ gained weight over 4 months. In both of the patients $(n=2)$ with raised BP the readings came back to normal after the intervention. One $(n=1)$ patient with normal BP at the baseline had high blood pressure following intervention.

Conclusion. Our service evaluation projects revealed that majority of the patients on the ward had deranged metabolic parameters such as increased BMI, abnormal blood tests and high BP.

Following our intervention more than a half of the patients lost weight whilst other half gained weight during the period of observation, which we suspect is associated with significant physical activity restrictions during the coronavirus pandemic. In both 
patients with high $\mathrm{BP}$ at the baseline it has improved following the intervention.

We would continue implementing healthy weight interventions on the ward and across the unit.

Diabetes care in an acute psychiatric inpatient setting: a logic model for service delivery

Zoe Goff ${ }^{1 \star}$, Allan House ${ }^{2}$, Elspeth Guthrie ${ }^{2}$, Hannah Weston ${ }^{3}$ and Laura Mansbridge ${ }^{3}$

${ }^{1}$ Leeds and York Partnership Foundation Trust, University of Leeds;

${ }^{2}$ University of Leeds and ${ }^{3}$ Leeds and York Partnership Foundation

Trust

${ }^{\star}$ Corresponding author.

doi: 10.1192/bjo.2021.847

Aims. To develop a logic model that illustrates the steps needed to develop an effective intervention for diabetes management in a psychiatric inpatient setting, as the point of admission to a psychiatric inpatient unit may present as an opportune time for improving diabetes care.

Method. We undertook (i) a survey of diabetes care among inpatients in a Mental Health Trust in England, comparing care to the National Health Service (NHS) Core National Diabetes Standards (ii) interviews with key clinical staff to understand challenges in delivering good diabetes care (iii) a review of current UK guidance on standards for diabetes care. On the basis of the findings we developed an initial logic model for service delivery.

Result. Among 163 inpatients reviewed, 44 (27\%) had a diagnosis of diabetes, and only 3 (7\%) had all three National Institute for Health and Care Excellence (NICE) treatment targets within range. Staff identified needs for regular training, better understanding of roles in shared care, and good quality IT support. We developed a logic model that illustrates the steps needed to develop an effective intervention for diabetes management in a psychiatric inpatient setting.

Conclusion. Admission to a psychiatric inpatient setting provides an opportunity in which diabetes care may be optimised. The quality and understanding of diabetes care will need to be enhanced if this opportunity is to be exploited.

Evaluation of a specialist service model for treating body dysmorphic disorder (BDD): application of National Institute for Health and Clinical Excellence Guidelines for BDD (NICE, 2006)

Anusha Govender

South West London \& St George's Mental Health NHS Trust

doi: 10.1192/bjo.2021.848

Aims. Body dysmorphic disorder (BDD) is still poorly recognised with a dearth of research into treatment. The OCD/BDD Service (South West London St. George's Mental Health NHS Trust) is a specialist service offering treatment for BDD using the National Institute for Health and Clinical Excellence (NICE) stepped care model for BDD as a basis for service provision.

This is the only known study to date to evaluate the implementation of the NICE guidelines recommended treatment for BDD in clinical practice. Furthermore, a sample of patients and clinicians were interviewed to elicit their evaluation of treatment.

Method. A total of 48 patients with a primary diagnosis of BDD who were offered treatment between 2006 and 2018 were identified. Questionnaires routinely completed at time of assessment were Yale Brown Obsessive Compulsive Scale for BDD (YBOCS-BDD); Montgomery-Asberg Depression Rating Scale (MADRS); Beck Depression Inventory (BDI) and Sheehan Disability Scale (SDS). Assessments were conducted by clinicians with expertise in BDD. Clinical data including risks and sociodemographic information were collated and analysed. Data were examined with intention to treat analysis. Thematic Analysis (TA) was used to analyse data from semi-structured interviews conducted with ten clinical staff and seven patients regarding their experiences of treatment. Qualitative data were coded and themes identified.

Result. Clinical data at assessment indicated impaired functioning plus high risks and substance misuse. There was a higher percentage of females (58\%); average duration of BDD was 19.23 years. Clinical outcomes indicated significant improvements in the total sample from baseline on measures of BDD, depression and functioning $(p=0.001)$. Patients described their progress in terms of living skills, social interactions and quality of life. The main themes identified included the significance of the therapeutic relationship expressed by both patients and clinicians; the lack of early intervention and knowledge of BDD in healthcare.

Conclusion. Current recommendations for treating BDD were found to be beneficial overall. However, there are patients who are non-responders and including experiences of patients' and clinicians' perspectives provided valuable insights into other options for treatment which are lacking and could enhance current recommendations where $\mathrm{CBT}$ and medication have not enabled progress. The young onset age with long duration, highlights the need for developing awareness of BDD so that it is not a hidden disorder.

\section{A service evaluation of the use and outcomes of} inpatient detoxification for the treatment of alcohol and opiate dependence within a community addictions service

\author{
Harry Griffin*, Natasha Rishi and Mike Kelleher \\ Lambeth Drug and Alcohol Service \\ ${ }^{*}$ Corresponding author.
}

doi: 10.1192/bjo.2021.849

Aims. The 2012 Health and Social Care Act transferred Addictions commissioning from the NHS to local authorities, leading to cuts of up to $30-50 \%$ of budgets and having the greatest impact on inpatient detox services. In a system with such limited capacity, effectively triaging access to detox services and optimising the efficacy of each detox has become increasingly important. NICE offers limited guidelines to assist with making these decisions, focused on assessing the severity of dependence and risk, but provides little detail on specific predictors of success. Our aim is to evaluate the nature of cases referred for inpatient alcohol or opiate detox and their treatment outcomes. This will help develop our understanding of the factors which influence achieving abstinence, and inform future decision-making regarding suitability for inpatient detox and post-detox planning. Conclusions will form part of a review of the local alcohol care pathway guidelines.

Method. A retrospective case note review of all inpatient detox admissions between April 2019-March 2020 ( $\mathrm{n}=113$ patients) is being undertaken. Our data collection tool extracts quantitative and qualitative data based on criteria from Alcohol use disorders (NICE, 2017), Opiate detoxification (NICE, 2019) and local pathway guidelines. 